\title{
Adaptive Image Enhancement Algorithm with Complex Background
}

\author{
Zhang Pai*
}

Department of Information Engineering, Tang Shan College, Tang Shan, 063000, P.R. China

\begin{abstract}
How to capture and enhance the target from an image with complex texture and edge features is studied in this paper. For the traditional image enhancement methods can not split different kinds of the textures and edges of an image efficiently, a novel adaptive enhancement algorithm based on nonsubsampled contourlet transform (NSCT) is projected. Firstly, by using the direct spatial multiplication of NSCT coefficients of each same directional subband at adjacent highpass scales, the inter-scale spaces are constructed and the optimal geometrical directions of each matrix region are calculated in the proposed spaces. Experimental results show that, in NSCT domain, the geometrical direction information which locates in the inter-scale spaces can represent the different detail features exactly of an image. Therefore, the optimal geometrical direction information as a rule to determine what kind of edge and texture features would be captured and enhanced is employed. After comparing with existing enhancement method, the proposed approach achieves better results, not only clearly identifies target edges and textures from complex background, but also enhances them with better visualization.
\end{abstract}

Keywords: Complex background, Image enhancement, NSCT, Optimal geometrical direction information.

\section{INTRODUCTION}

Image enhancement is to improve the interpretability or perception of information in images for human viewers, or to provide 'better' input for other automated image processing techniques. In this paper, a type of image is discussed whose targets possess complex edges and high contrast texture features while the background also has complex edges but lower contrast texture features. The aim is to capture and enhance the targets accurately from the images. Most of traditional enhancement methods [1-3] can enhance weak edges and features in an image while restraining the noise and keeping the strong edges, but they are invalid in identifying and enhancing the edges or textures from objective region in an image with complex background.

To overcome the limitation of existing enhancement methods and make full use of the test image features, we come up with a novel adaptive enhancement algorithm based on nonsubsampled contourlet transform (NSCT) [4]. As a shift-invariant version of the contourlet transform [5], NSCT provides an efficient directional multiresolution image representation. In NSCT domain, each of highpass directional subbands shows a high degree of directionality and anisotropy. Taking into account the coefficients' dependencies across scale in multiresolution decomposition and the features of test images, we used the direct spatial multiplication of NSCT coefficients of each same directional subband at adjacent scales to construct the inter-scale spaces and implemented two concrete steps in the constructed interscale spaces to distinguish target's edges and textures from

*Address correspondence to this author at the Department of Information Engineering, Tang Shan College, Tang Shan, 063000, P.R. China;

Tel: 13933958293; E-mail: raindrop_pai@qq.com backgrounds. Firstly, based on the idea of bandelet transform [6], we computed the optimal geometrical information of sub-squares (of matrix region) in constructed inter-scale spaces. This geometrical information can create a full distinction between high contrast edge and texture features and lower contrast edge and texture features of the source image. Then, in terms of the geometrical directions, an adaptive enhancement method was employed to preserve and enhance the goals, while weakening the background.

In this paper, the motivation is to project a new enhancement algorithm which has ability in capturing and enhancing the target with high contrast features in comparison with the background of source images. Experimental results show the availability of our approach.

\section{PRINCIPLE OF NSCT AND THE CONSTRUCTION OF THE OPTIMAL GEOMETRICAL INFORMATION IN ITS INTER-SCALE SPACES}

\subsection{The Principle of NSCT}

In the foremost contourlet transform, downsamplers and upsamplers are presented in both the laplacian pyramid (LP) and the directional filter banks (DFB). Thus, it is not shiftinvariant, which causes pseudo-Gibbs phenomenon around singularities. NSCT is an improved form of contourlet transform.

In contrast with contourlet transform, nonsubsampled laplacian pyramid (NLP) structure and nonsubsampled directional filter banks (NDFB) are employed in NSCT. The NLP structure is achieved by using two-channel nonsubsampled 2D filter banks. The NDFB is achieved by switching off the downsamplers/upsamplers in each two- 
channel filter bank in the DFB tree structure and upsampling the filters accordingly.

As a result, NSCT is shift-invariant and leads to better frequency selectivity and regularity. Fig. (1) shows the decomposition framework of NSCT.

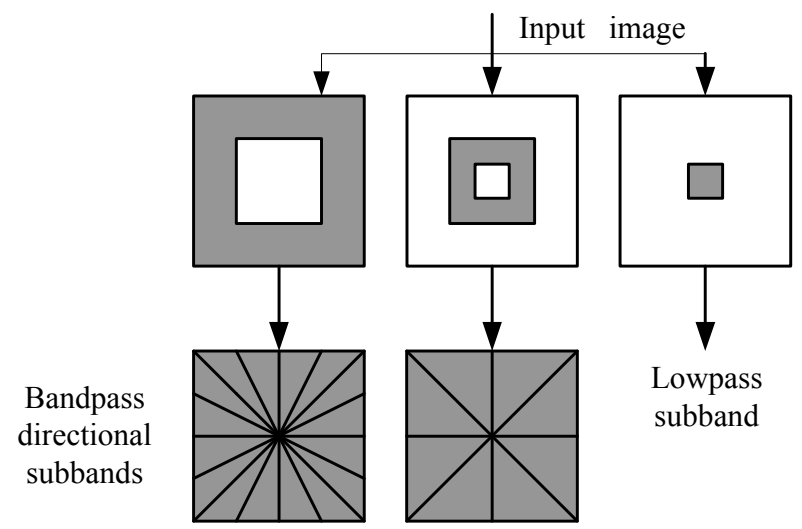

Fig. (1): Decomposition framework of NSCT.

In contrast with contourlet transform, nonsubsampled laplacian pyramid(NLP) structure and nonsubsampled directional filter banks (NDFB) are employed in NSCT. The NLP structure is achieved by using two-channel nonsubsampled 2D filter banks. The NDFB is achieved by switching off the downsamplers/upsamplers in each twochannel filter bank in the DFB tree structure and upsampling the filters accordingly. As a result, NSCT is shift-invariant and leads to better frequency selectivity and regularity. Fig. (1) shows the decomposition framework of NSCT.

\subsection{The Construction of the Optimal Geometrical Information in its Inter-Scale Spaces}

In wavelet domain, the direct correlation of wavelet coefficients was used by $\mathrm{Xu}$ and co-workers to selectively denoise images in an edge-oriented manner [7]. It has been observed that by multiplying the adjacent scales edges could be sharpen while diluting noise. This paper proposes an NSCT based edge detection scheme by scale multiplication. The NSCT coefficients coming from same directional subbands at two adjacent scales are multiplied as a product function. We define the function as follows:

$$
N_{-} \text {Corr }_{l, l+1}^{d}=N_{l}^{d} \times N_{l+1}^{d}
$$

Here, $\quad N_{-}$Corr $_{l, l+1}^{d}$ represents the product data of NSCT coefficients in the $d$ direction at $l$ and $l+1$ scales. $N_{l}^{d}$ and $N_{l+1}^{d}$ are the NSCT coefficients of d direction at adjacent 1 and $1+1$ scales, respectively, where $l(l \in[1, L-1], L$ is the total number of scales) is the number of scales involved in the direct multiplication and $d(d \in[1, D], D$ is the total number of directions) is the number of directions in corresponding scales.
NSCT is shift-invariant such that each coefficient of the transform subbands corresponds to that of the original image in the same location and the $N_{-}$Corr $_{l, l+1}^{d}$ of each adjacent scales has the same property. On comparing with the coefficients of subbands in each single scale, $N_{-} \operatorname{Corr}_{l, l+1}^{d}$ over two adjacent scales sharpens and enhances major edges while suppressing small sharp features. Fig. (2) as an illustration shows the effectiveness of $N_{-} \operatorname{Corr}_{l, l+1}^{d}$ in representing the locations of edges and other significant features of images.
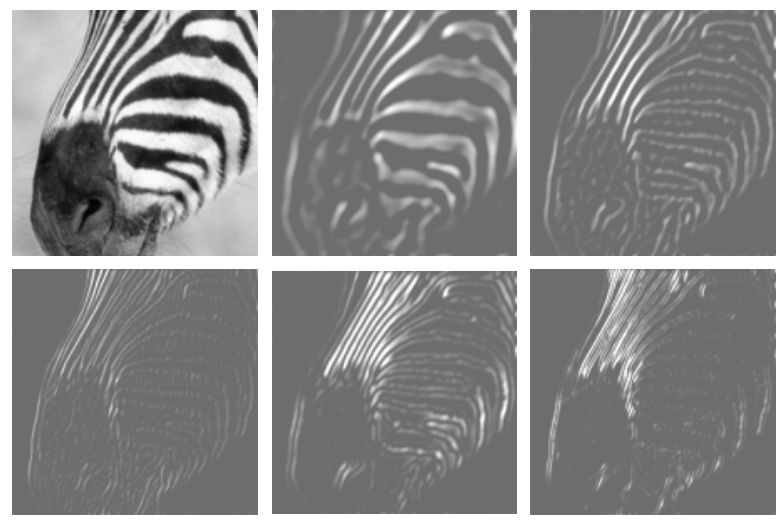

Fig. (2): An illustration of NSCT coefficients in the same direction(where ) at several single scales(where ) and coefficient products at corresponding adjacent scales of an "horse" image. From top left to bottom right: Original image; $N_{2}^{1} ; N_{3}^{1} ; N_{4}^{1}$; $N_{-} \operatorname{Corr}_{2,3}^{1} ; N_{-} \operatorname{Corr}_{3,4}^{1}$.

From Fig. (2), we can observe that, in each coefficient space, non-zero data represent the singularities of source image. In $N_{l}^{d}$ spaces, too many edge and texture features are presented that can not distinguish them in an efficient way. In $N_{-}$Corr $_{l, l+1}^{d}$ spaces, only significant edges and textures are preserved while small edges and regular parts of source image are eliminated. Therefore, the geometrical information in $N_{-}$Corr $_{l, l+1}^{d}$ spaces is more effective and tractable.

Next, we need to exploit an efficient approach to distinguish different edge and texture features in each $N_{-} \operatorname{Corr}_{l, l+1}^{d}$ space. Firstly, we would like to introduce a brief review of the bandelet transform. The bandelet approximation scheme, introduced in [6], takes advantage of geometric image regularity by removing the redundancy of a

warped wavelet transform by performing a bandeletization which is defined as reordering the $2 \mathrm{D}$ wavelet coefficients and then performing a 1D wavelet transform.

The classical tensor wavelet transform of an image is the decomposition of the latter on an orthogonal basis formed by the translation and dilation of three mother wavelets $\left\{\psi_{H}, \psi_{V}, \psi_{D}\right\}$ for the horizontal, vertical and diagonal directions. Once the wavelet transform is found, the quadtree is computed by dividing the image into dyadic squares with 
variable sizes (refer to [6] for more information on computing the quadtree). For each square in the quadtree the optimal geometrical direction is computed by the minimization of a lagrangian (refer also to [6]). Then, a projection of the wavelet coefficients along the optimal direction is performed [6]. Finally, a 1D discrete wavelet transform is carried on the projected coefficients. For each highpass scale of the wavelet transform, the quadtree and a zoom on the orientation of the linear flow on each dyadic square are required. Notice that the quadtree segmentation performs very well.

Based on the idea of bandelet transform, we computed the optimal geometrical direction of each square with a fixed size in the proposed $N_{-} \operatorname{Corr}_{l, l+1}^{d}$ spaces. The best direction is the one that leads to the best compressed representation after the bandeletization. If there is no preferential orientation in the square $\mathrm{S}$, then it is better not to perform any bandeletization. In this case, we set direction is equal to NULL in the present square S. Fig. (3) as an illustration shows the optimal geometrical information of each square $\mathrm{S}$ in one of the $N_{-}$Corr $_{l, l+1}^{d}$ spaces of the part of horse image.

For the square whose direction is not equal to NULL, we now perform the $1 \mathrm{D}$ discrete reordering of the sampling location. This is done by projecting the sampling location along geometrical direction $\mathrm{d}$ and sorting the resulting 1D points from left to right. The obtained 1D numbering of the sampling points defines a $1 \mathrm{D}$ discrete signal $f_{d}$, and then performs a 1D discrete wavelet transform of $f_{d}$. We denote by $\left\{b_{k}\right\}$ the coefficients of the $1 \mathrm{D}$ wavelet transform of $f_{d}$. From $\left\{b_{k}\right\}$, one can see that the high wavelet coefficients corresponding to most of edge information are gathered, and this property provides convenience in the following processing.

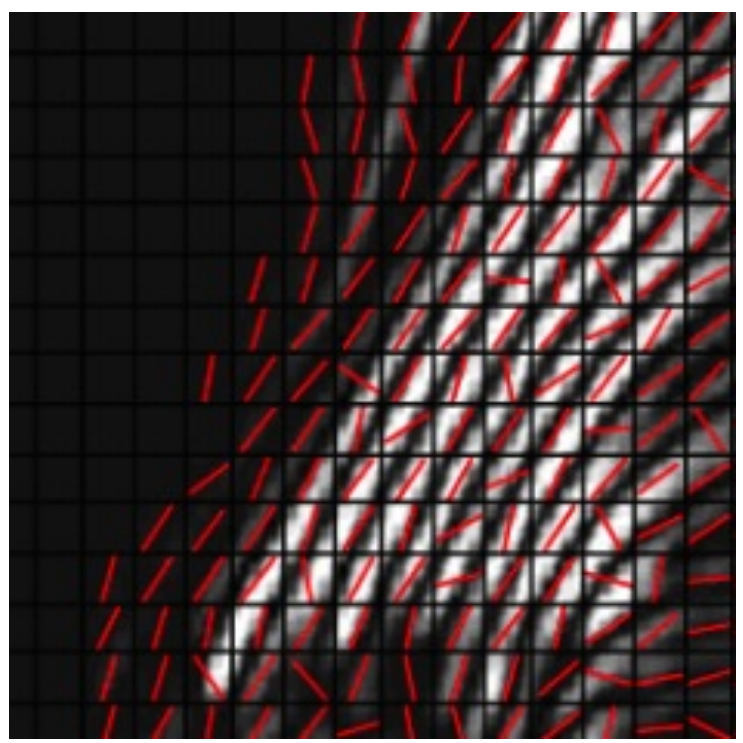

Fig. (3): The optimal geometrical information of each square $\mathrm{S}$ in one of the $N_{-} \operatorname{Corr}_{l, l+1}^{d}$ spaces of the part of "horse " image.

\section{IMAGE ENHANCEMENT ALGORITHM}

\subsection{The Effectiveness of Image Edges Representation in the Inter-Scale Space}

When encountering a type of images whose targets are possessed of complex strong edges and high contrast texture features while the background also has complex strong edges but lower contrast texture features, we are inclined to capture and enhance the target from complex background. Traditional enhancement algorithms lose efficacy in solving the problem. To make full use of the own features of this type of images while processing them has become significant.

Refer to [7] pointed out that the steep regions of source image yield bigger coefficient amplitudes in frequency domain while the smooth region is corresponding with small coefficient amplitudes.

In this section, we would like to explore the properties of different kinds of edge and texture features representation in proposed space. The "horse" image is still taken as a test image decomposed by NSCT in several scales. Fig (4) shows each inter-space constructed by corresponding $N_{-} \operatorname{Corr}_{l, l+1}^{d}$ (where $l=3, l+1=4$ and the total direction num is set as four which is the same in two adjacent scales).
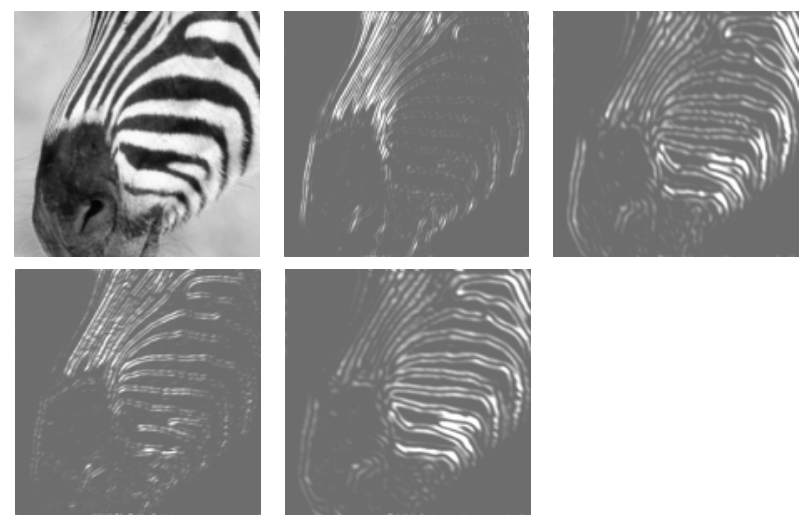

Fig. (4): An illustration of inter-scale spaces of all directions. From left to right: Original image; $N_{-} \operatorname{Corr}_{3,4}^{1} ; \quad N_{-} \operatorname{Corr}_{3,4}^{2}$; $N_{-} \mathrm{Corr}_{3,4}^{3} ; N_{-} \mathrm{Corr}_{3,4}^{4}$.

From Fig. (4), it can be observed that the side face of horse has high contrast textures but not so strong as textures of the front face are transformed into 'weak' edges in the $N_{-} \operatorname{Corr}_{l, l+1}^{d}$ spaces, while the textures of the front face are transformed into 'strong' edges and the textures of the nose are suppressed in the $N_{-} \operatorname{Corr}_{l, l+1}^{d}$ spaces. Hence, it can be concluded that the weak edges in $N_{-}$Corr $r_{l, l+1}^{d}$ space are the representation of texture features with high contrast in source image.

Now, to orientate weak edges accurately in $N_{-} \operatorname{Corr}_{l, l+1}^{d}$ space becomes an important issue. In NSCT domain, the 
strong edges correspond to those pixels with big-value coefficients in all subbands. The weak edges correspond to those pixels with big-value coefficients in some directional subbands but small-value coefficients in other directional subbands within the same scale. The noises correspond to those pixels with small-value coefficients in all subbands. (refer also to [8]).This observation is also effective in $N_{-} \operatorname{Corr}_{l, l+1}^{d}$ spaces. Depending on the geometrical direction information of each fixed square region in $N_{-} \operatorname{Corr}_{l, l+1}^{d}$ spaces, we exploit a new approach by adding up to the number of geometrical directions which are unequal to NULL to determine the class of edges in the present fixed square region. Then, repeat the previous steps to detect other sub-regions. The detailed description will be taken in the next section.

\subsection{The Implement Steps of the Enhancement Algorithm}

In this section, the implement steps of proposed enhancement algorithm can be divided into four different stages with reference to Fig. (5).

\section{Stage I}

1) Make the source image via NSCT and illustrate it. The mapping is assumed to be of the form: $x^{0} \rightarrow\left\{N_{1}, N_{2}, \cdots N_{L}, x_{L}\right\}$. Where, $N_{l}$ is the detail image at level $l$ and $x_{L}$ is the approximation at the coarsest level $L$. Then, $N_{l}, l=1,2 \cdots L$ is further separated into subbands according to its orientation, namely, $N_{l}=\left\{N_{l}^{1}, N_{l}^{2}, \cdots, N_{l}^{D}\right\}$ representing $\mathrm{D}$ directions. Here, the number of directional subbands at all high-frequency levels is the same with each other, which is for preparation of constructing the inter-scale coefficient product spaces in the same directional subband across adjacent levels.

2) By using the direct spatial multiplication of NSCT coefficients of each same directional subband at adjacent scales to construct the inter-scale spaces. $N_{-} \operatorname{Corr}_{l, l+1}^{d}$ represents the product data of NSCT coefficients in $d$ direction at $l$ and $l+1$ scales, where $l(l \in[1, L-1], L$ is the total number of scales) is the number of scales involved in the direct multiplication and $d(d \in[1, D], D$ is the total number of directions) is the number of directions in corresponding scales. This is defined as previous section.

3) Divide $N_{-}$Corr $_{l, l+1}^{d}$ into subregions (of $4 \times 4$ matrix region) which can be denoted as $\left\{N_{-} \operatorname{Corr}_{l, l+1}^{d}(1), N_{-} \operatorname{Corr}_{l, l+1}^{d}(2), \cdots, N_{-} \operatorname{Corr}_{l, l+1}^{d}(P)\right\} \cdot P$ is the total number of subregions. Then, we perform "bandazation" to calculate the optimal geometrical directions of $N_{-} \operatorname{Corr}_{l, l+1}^{d}(p)$ and define $\theta_{l, l+1}^{d}(p)$ as representation of direction information of each $p$ square region, especially, $\theta_{l, l+1}^{d}(p)=\operatorname{Inf}$ represents non geometrical information in $N_{-} \operatorname{Corr}_{l, l+1}^{d}(p)$ region. Here, we define a new variable
$S_{l, l+1}^{d}(p)$ to divide $\theta_{l, l+1}^{d}(p)$ into two categories, which is stated as follows:

$S_{l, l+1}^{d}(p)= \begin{cases}1 & \theta_{l, l+1}^{d}(p) \neq \operatorname{Inf} \\ 0 & \text { else }\end{cases}$

where $l \in[1, L-1], d \in[1, D], p \in[1, P]$.

\section{Stage II}

1) In term of the geometric information features pixel by pixel of the NSCT coefficients demonstrated in section 3.1, we observe that in the same inter-scales, the weak edges probably exist in the sub-region whose $\theta_{l, l+1}^{d}(p)=\operatorname{Inf}$ at a part of directional subbands while at others, $\theta_{l, l+1}^{d}(p) \neq \operatorname{Inf}$, the strong edges correspond to $\theta_{l, l+1}^{d}(p) \neq \operatorname{Inf}$ through the whole directional subbands, the small edges and smooth regions correspond to $\theta_{l, l+1}^{d}(p) \neq \operatorname{Inf}$ at most of directional subbands.

These properties prompt us the total number of path regions whose $\theta_{l, l+1}^{d}(p) \neq \operatorname{Inf}$ at all directional subbands in present inter-scale can be used as a rule to classify the edges. Equation (3) is a statement of the concrete rule.

$$
\text { edge_style }= \begin{cases}\text { small edge } & 0 \leq \sum_{d=1}^{D} S_{l, l+1}^{d}(p)<\frac{D}{2}-1 \\ \text { weak edge } & \frac{D}{2}-1 \leq \sum_{d=1}^{D} S_{l, l+1}^{d}(p) \leq D-1 \\ \text { strong edge } & \sum_{d=1}^{D} S_{l, l+1}^{d}(p)=D\end{cases}
$$

The thresholds of determining the edge styles are adopted by the test of numerous experiments. We further investigated to generate a map composed of 0 or 1 as the representation of the different type of edges.

$$
\operatorname{map}_{l, l+1}(p)= \begin{cases}1 & \text { weak edge } \\ 0 & \text { else }\end{cases}
$$

2) Here are two confusing issues: Firstly, owing to the complexity and irregularity of background in source images, weak edges may also exist in the region of $N_{-} \operatorname{Corr}_{l, l+1}^{d}$ spaces which correspond to the source background regions. This will cause bad accuracy in capturing the target's regions. Secondly, the strong edges are both in the target's and the background's region of source images, and how to identify strong edges of the target region is another issue we need to solve.

To settle these difficulties, we need to consider the features of source images once again. Firstly, in source images, owing to the fact that texture features of background often possess lower contrast and not so steep, their corresponding sub-regions with weak edges in inter-scale would be scattered and independent. Secondly, the target's strong edges are along with its texture features to compose an integral goal. 
Hence, we affirm that the strong edge regions which surround the weak edge regions in each inter-scale space are the corresponding edges belonging to the target in source image.

In terms of these two analyses, we produce an operator which consists of $3 \times 3$ kernels of the form $W=\left[\begin{array}{lll}1 & 1 & 1 \\ 1 & 1 & 1 \\ 1 & 1 & 1\end{array}\right]$ and

use it to operate the map matrix (formula 5). The function of this operator is to reset the centre element value, which can coincide with most of the neighbors around it in the present $3 \times 3$ region. This implementation can remove the single region with singularity in the inter-scale spaces, meanwhile preserving the objective strong edge regions. The following formula is a statement of the concrete rule of the operator.

$\operatorname{map}_{l, l+1}(p)= \begin{cases}1 & \text { if } \sum_{\substack{i \in[-1,1] \\ j \in[-1,1]}} \operatorname{map}(p+i, p+j) \geq 6 \\ 0 \quad \text { else } & \end{cases}$

The element values of new map matrix can represent the belongings of corresponding sub-regions exactly in interscale spaces. Now, we achieve in capturing the target's region in the source. In the next stage, we would like to exploit an effective approach to enhance the objective region.

\section{Stage III}

1) The $\operatorname{map}_{l, l+1}(p), \quad \theta_{l, l+1}^{d}(p), \quad l \in[1, L-1], \quad$ and $d \in[1, D], p \in[1, P]$ are used as the information to operate NSCT corresponding directional subbands in previous $l$ scale $l \in[1, L-1]$. For the finest scale $(l=L)$ represents most of the strong edges feature, we are not taking it into account here. In order to highlight the objective region, an operation is performed by enhancing the target meanwhile weakening the background. At each highpass directional subband in NSCT domain, by using $\operatorname{map}_{l, l+1}(p), \theta_{l, l+1}^{d}(p)$ as the rule, the corresponding sub-regions can be determined whether to enhance or not. The concrete rule is shown as follows:

$$
\begin{aligned}
& \text { sub-region }{ }_{l}^{d}(p)=\left\{\begin{array}{ll}
\text { enhance } & \operatorname{map}_{l, l+1}(p)=1 \\
\text { weaken } & \operatorname{map}_{l, l+1}(p)=0
\end{array},\right. \\
& p \in[1, P] ; d \in[1, D]
\end{aligned}
$$

2) To provide an effective way in enhancing the objective region, we propose a new enhancement method named BD$1 \mathrm{D}$ algorithm by considering $\left\{b_{k}\right\}$ (has been mentioned in previous section) as each sub-region's data. The BD-1D algorithm is implemented as follows: firstly, the threshold of each $\left\{b_{k}\right\}$ is calculated respectively in formula (7). Then, the coefficients greater than the threshold are regarded as the high coefficients which represent the edges information in the $\left\{b_{k}\right\}$, we enhance them with a suitable weight (here, it is set to 3 ). The corresponding formula is shown as follows:

$$
\begin{gathered}
T=\frac{\operatorname{median}(|b(i, j)|)}{0.6745}, b(i, j) \in\left\{b_{k}\right\} \\
b(i, j)=\left\{\begin{array}{ll}
b(i, j) \times 3 & b(i, j)>T \\
b(i, j) & b(i, j)<T
\end{array}, b(i, j) \in\left\{b_{k}\right\}\right.
\end{gathered}
$$

here, $b(i, j)$ represents each coefficient of $\left\{b_{k}\right\}$.

\section{Stage IV}

Perform inverse 1D wavelet transform on each $\left\{b_{k}\right\}$. Then, by using the processed coefficients reconstruct the enhanced image via inverse NSCT.

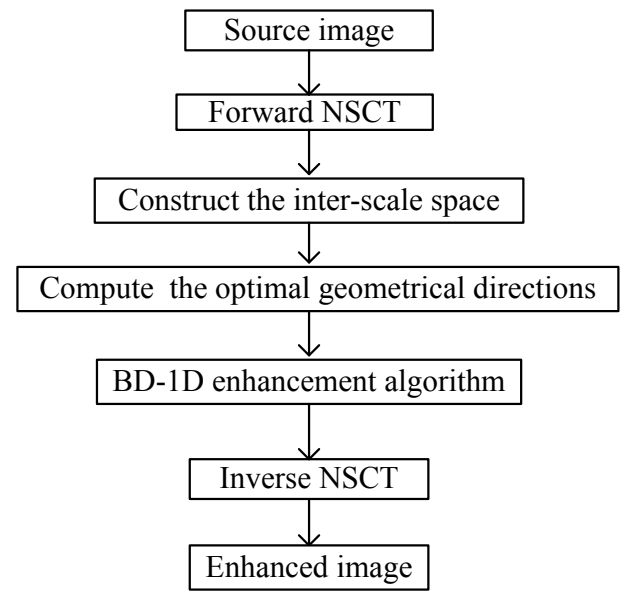

Fig. (5): Schematic diagram of proposed fusion algorithm.

Intelligent sketch technology is based on the study of design thinking, sketches behavior, its characteristics, the paper Sketch design technology and computer support, each of which has its own strengths and weaknesses [8]. It will be a very useful work if their advantages are combined together. Especially, now the CAD technology which orients the detailed design stage has developed perfectly. It can guarantee the support and assistance of the computer for the entire design process, consistently unifying the entire design process. Moreover, the outstanding characteristics of the computer itself are bound to raise the level of efficiency and design at the early design stage. Especially, it can enhance the sketch design innovation performance through smart innovation and technology. The key to intelligent sketch technology is in-depth understanding of the draft plan to the behavior. Sketching behavior and design thinking are closely related. The sketches capture inherent human behavior thinking outside or concrete which is a necessary extension of the visual image of the designer's perception. Intelligent sketch technology for computer support, according to the characteristics of the computer itself, can be roughly divided into four categories: Natural interaction behavior smart sketch; Sketch of expression and freedom of freehand sketch input; Design intent capture and sketch recognition; and sketch-based geometric modeling. Fig. (1) shows the 
computer support hand-painted ceramic pattern sketches technology framework.

\section{EXPERIMENTAL RESULTS}

In this section, two typical images with complex edge and texture features are taken as the experimental images. Our aim is to capture and enhance the target from background accurately. We compared the enhancement results by the proposed algorithm with those by the BD-1D algorithm in wavelet transform and enhancement algorithm in NSCT domain.

In the first experiment, the input is a butterfly in the littery grass as shown in Fig. (6).
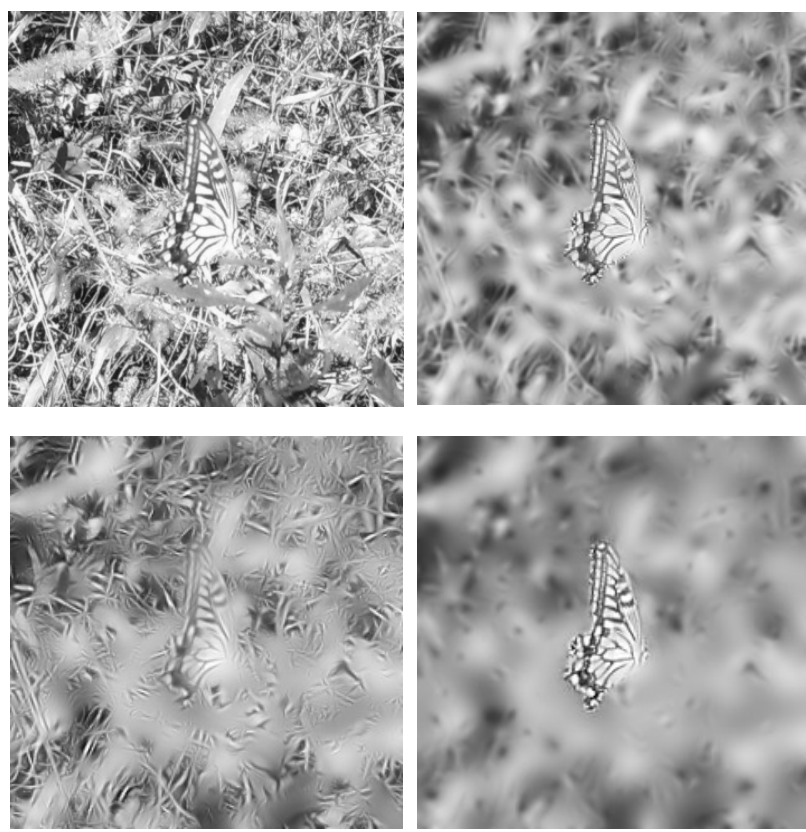

Fig. (6) "Butterfly" image enhancement results. From top to bottom: Original image; enhanced image based BD-1D in wavelet domain; Enhanced image based NSCT enhancement algorithm; Enhanced image based the proposed algorithm.

From Fig. (6), it can be observed that, in the enhanced image of BD-1D enhancement algorithm in wavelet domain method, some of the edges and textures from background are preserved and enhanced unexpectedly, which causes the final experimental result to be unsatisfactory in visual observation. The reason is that, the wavelets lack the important feature of directionality and hence, they are not efficient in retaining textures and fine details of images in wavelet domain. NSCT enhancement algorithm does better jobs in enhancing the weak edges in the textures and distinguishing weak edges from noises, but losing efficacy in capturing the objective region and hence its enhanced result is not excellent in visual appearance either. Our experiments show that the proposed approach outperforms other enhancement methods in visual effect. Fig. (7), as a description of a coccinella septempunctata in the cereus, will give another strong example.
From Fig. (7), we can observe that the proposed algorithm removes most of the edge and texture features belonging to the background and also performs excellent result in enhancing the objective region in the test image when compared with other two listed methods.
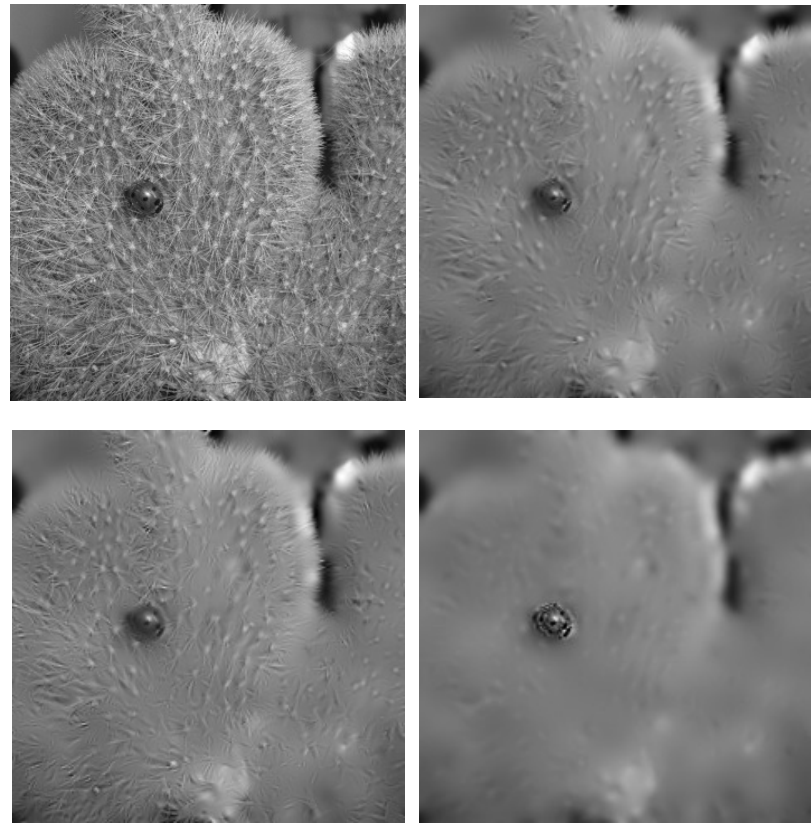

Fig. (7): "Cactus" image enhancement results. From top to bottom: Original image; enhanced image based BD-1D in wavelet domain; Enhanced image based NSCT enhancement algorithm; Enhanced image based the proposed algorithm.

\section{CONCLUSION}

In this paper, we proposed a new algorithm in capturing and enhancing the objective region from an image with complex texture and edge features. The approach based on NSCT, by using the coefficient products of each inter-scale and the corresponding geometrical direction information in the proposed spaces, we can detect the location of the target accurately from source image. Then, we project a BD-1D algorithm to enhance the target. The simulation results indicated that the proposed method is visually superior to others in preserving and enhancing edges and textures of target.

\section{CONFLICT OF INTEREST}

The author confirms that this article content has no conflict of interest.

\section{ACKNOWLEDGEMENTS}

Declared none.

\section{REFERENCES}

[1] A. Laine, J. Fan, and W. Yang, "Wavelets for contrast enhancement of digital mammography", IEEE Engineering in Medicine and Biology, pp. 536-550, 1995. 
[2] S. Dippel, M. Stahl, R. Wiemker, and T. Blaffert, "Multiscale contrast enhancement for radiographies: Laplacian pyramid versus fast wavelet transform", IEEE Transactions in Medical Imaging, vol. 21, no. 4, pp. 343-353, 2002.

[3] K. V. Velde, "Multi-scale color image enhancement", Proceedings of IEEE International Conference on Image Processing, vol. 3, pp. 584-587, 1999.

[4] A. L. Cunha, J. Zhou, and M. N. Do, "The nonsubsampled contourlet transform: theory, design and applications", IEEE Transactions on Image Processing, vol. 15, pp. 3089-3101, 2006.

[5] M. N. Do and M. Vetterli, "The contourlet transform: An efficient directional multiresolution image representation", IEEE Transactions on Image Processing, vol. 14, pp. 2019-2106, 2005.
[6]

G. Peyr, and S. Mallat, "Surface compression with geometric bandelets," ACM Transactions on Graphics (TOG), vol. 24, no.3, pp. 601-608, 2005.

[7] Y. Xu, J. B. Weaver, D. M. Healy, and J. Lu, "Wavelet transform domain filters: A spatially selective noise filtration technique", IEEE Transactions on Image Processing, vol. 3, pp. 747-758, 1994.

[8] J. Zhou, A. L. Cunha and M. N. Do, "Nonsubsampled contourlet transform: Construction and application in enhancement", Proceedings on IEEE International Conference on Image, vol. 1, pp. 469, 2005.

(C) Zhang Pai.; Licensee Bentham Open.

This is an open access article licensed under the terms of the Creative Commons Attribution Non-Commercial License (http://creativecommons.org/licenses/by-nc/4.0/) which permits unrestricted, non-commercial use, distribution and reproduction in any medium, provided the work is properly cited. 